\title{
Specifying Avalon Objects in Larch
}

\author{
Jeannette M. Wing ${ }^{1}$ \\ Invited Paper \\ Department of Computer Science \\ Carnegie Mellon University \\ Pittsburgh, PA 15213
}

\begin{abstract}
This paper gives a formal specification of three base Avalon/C++ classes: recoverable, atomic, and subatomic. Programmers derive from class recoverable to define persistent objects, and from either class atomic or class subatomic to define atomic objects. The specifications, written in Larch, provide the means for showing that classes derived from the base classes implement objects that are persistent or atomic, and thus exemplify the applicability of an existing specification method to specifying "nonfunctional" properties. Writing these formal specifications for Avalon/C+H's built-in classes has helped clarify places in the programming language where features interact, make explicit unstated assumptions, and make precise complex properties of objects.
\end{abstract}

\section{Introduction}

Formal specification languages have matured to the point where industry is receptive to using them and researchers are building tools to support their use. People use these languages for specifying the inputoutput behavior, i.e., functionality, of programs, but have largely ignored specifying a program's "nonfunctional" properties. For example, the functionality of a program that sorts an array of integers might be informally specified as follows: given an input array $A$ of integers, an array $B$ of integers is returned such that B's integers are the same as A's, and B's are arranged in ascending order. Nothing is said about the program's performance like whether the algorithm for sorting should be $O(n)$ or $O\left(n^{2}\right)$. Performance is one example of a non-functional property. Other non-functional properties are degree of concurrency, reliability, and security.

In this paper, we demonstrate the applicability of formal specifications to the non-functional properties, persistence and atomicity. Atomicity, which subsumes persistence, requires that an object's state be correct in the presence of both concurrency and hardware failures. The correct behavior of these objects is fundamental to the correctness of the programs that create, access, and modify them. Section 2 describes in more detail the context in which atomic objects are used: fault-tolerant distributed systems. Sections 3, 4, and 5 present a concrete programming language interface to such objects and the formal specifications of this interface.

Section 6 summarizes the lessons learned from writing these specifications out formally. The results are extremely gratifying: they provide evidence that an existing specification method is suitable for describing a new class of objects; they validate the correctness of the design and implementation of a key part of an ongoing software development project; and not surprisingly, they demonstrate that the process of writing formal specifications greatly clarifies one's understanding of complex behavior. Finally, Section 7 concludes with remarks about current and future work.

\footnotetext{
'This research was sponsored in part by the Defense Advanced Research Projects Agency (DOD), ARPA Order No. 4976 (Amendment 20), under contract F33615-87-C-1499 monitored by the Avionics Laboratory, Air Force Wright Aeronautical Laboratories, Wright-Patterson AFB and in part by the National Science Foundation under grant CCR-8620027. The views and conclusions contained in this document are those of the author and should not be interpreted as representing the official policies, either expressed or implied, of the Defense Advanced Research Projects Agency or the US Govemment.
} 


\section{Background}

\subsection{Abstract Context: Transaction Model of Computation}

A distributed system runs on a set of nodes that communicate over a network. Since nodes may crash and communications may fail, such a system must tolerate faults; processing must continue despite failures. For example, an airline reservations system must continue servicing travel agents and their customers even if an airline's database is temporarily inaccessible; an automatic teller machine must continue dispensing cash even if the link between the ATM and the customer's bank account is down.

A widely-accepted technique for preserving data consistency and providing data availability in the presence of both concurrency and failures is to organize computations as sequential processes called transactions. A transaction is a sequence of operations performed on data objects in the system. For example, a transaction that transfers $\$ 25$ from a savings account, $S$, to a checking account, $C$, might be performed as the following sequence of three operations on $S$ and $C$ (both initially containing \$100):

$$
\begin{gathered}
\{S=\$ 100 \wedge C=\$ 100\} \\
\operatorname{Read}(S) \\
\operatorname{Debit}(S, \$ 25) \\
\operatorname{Credit}(C, \$ 25) \\
\{S=\$ 75 \wedge C=\$ 125\}
\end{gathered}
$$

In contrast to standard sequential processes, transactions must be atomic, that is serializable, transactionconsistent, and persistent. ${ }^{2}$ Serializability means that the effects of concurrent transactions must be the same as if the transactions executed in some serial order. In the above example, if two transactions, T1 and $T 2$, were simultaneously transferring $\$ 25$ from $S$ to $C$, the net effect to the accounts should be that $S$ $=\$ 50$ and $C=\$ 150$ (that is, as if $\mathrm{T} 1$ occurred before $\mathrm{T} 2$ or vice versa). Transaction-consistency means that a transaction either succeeds completely and commits, or aborts and has no effect. For example, if the transfer transaction aborts after the Debit but before the Credit, the savings account should be reset to $\$ 100$ (its balance before the transfer began). Persistence means that the effects of committed transactions survive failures. If the above transfer transaction commits, and a later transaction that modifies $\mathrm{S}$ or $\mathrm{C}$ aborts, it should be possible to "roll back" the state of the system to the previous committed state where $S=\$ 75$ and $C=\$ 125$.

It can be shown [17] that the atomicity of the entire system is guaranteeed if each object accessed within transactions is atomic. That is, each object is an instance of an abstract data type with the additional requirement that it must ensure the serializability, transaction-consistency, and persistence of all the transactions that use its operations. For example, as long as the bank account's Read, Debit, and Credit operations are implemented "correctly," then any set of transactions that access the account will be serializable, transaction-consistent, and persistent. The advantage of constructing a system by focusing

\footnotetext{
${ }^{2}$ Unfortunately, no standard terminology is used for the terms transaction-consistent and persistent. Transaction-consistent is sometimes called failure atomic, total, or simply atomic. Persistent is sometimes called recoverable, permanent, or resilient. In this paper, we use terminology consistent with Avalon terminology as published in [5].
} 
on individual objects instead of on a set of concurrent transactions is modularity: one need only ensure that each object is atomic to ensure the more global atomicity property of the entire system.

\subsection{Concrete Context: Avalon}

The Avalon Project [5], conducted at Carnegie Mellon University, provides a concrete context for this work. We have implemented language extensions to $\mathrm{C}++[16]$ to support application programming of fault-tolerant distributed systems. Avalon relies on the Camelot System [15], also being developed at $\mathrm{CMU}$, to handle operating-systems level details of transaction management, inter-node communication, commit protocols, and automatic crash recovery.

A program in Avalon consists of a set of servers, each of which encapsulates a set of objects and exports a set of operations and a set of constructors. A server resides at a single physical node, but each node may be home to multiple servers. An application program may explicitly create a server at a specified node by calling one of its constructors. Rather than sharing data directly, servers communicate by calling one another's operations. An operation call is a remote procedure call with call-by-value transmission of arguments and results. Avalon/ $\mathrm{C}++$ includes a variety of primitives (not discussed here) for creating transactions in sequence or in parallel, and for aborting and committing transactions. Each transaction is identified with a single process (thread of control).

Transactions in Avalon/C + may be nested. A subtransaction's commit is dependent on that of its parent; aborting a parent will cause a committed child's effects to be rolled back. A transaction's effects become permanent only when it commits at the top level. Each transaction has a unique parent, a (possibly empty) set of siblings, and sets of ancestors and descendants. A transaction is considered its own ancestor or descendant.

Avalon/C++ provides transaction semantics by requiring that all objects shared by transactions be atomic. The Avalon/C++ base hierarchy consists of three classes (Figure 2-1), each of which provides primitives for implementors of derived classes to ensure the "non-functional" properties of objects of the derived classes. Programmers derive from either class atomic or class subatomic to define their own atomic objects.

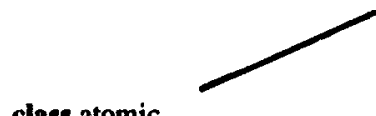

class atomic

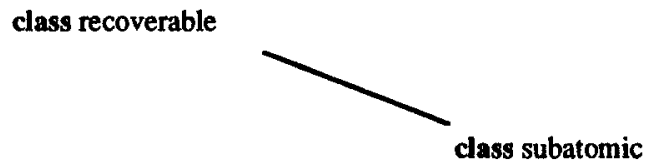

Figure 2-1: Inheritance Hierarchy of the Three Avalon/C++ Base Classes

In practice, sometimes it may be too expensive to guarantee atomicity at all levels of a system; instead it is often useful to implement atomic objects from non-atomic objects, those which guarantee only persistence. Programmers need only derive from class recoverable to define persistent objects.

In Avalon/C++ when a transaction commits, the run-time system assigns it a timestamp generated by a logical clock [12]. Atomic objects are expected to ensure that all transactions are serializable in the order of their commit timestamps, a property called hybrid atomicity [17]. This property is automatically ensured by two-phase locking protocols [6], used by objects derived from class atomic. However, 
additional concurrency can be achieved by taking the timestamp ordering explicitly into account, used by objects derived from class subatomic. The key difference between class atomic and class subatomic is that class subatomic gives programmers a finer-grained control over synchronization and crash recovery.

The main purpose of this paper is to give a formal specification of the interfaces of the three base Avalon/C++ classes, presented in Sections 3, 4, and 5. By specifying these interfaces, we provide the means for showing that classes derived from the base classes define objects that are persistent or atomic, i.e., the "non-functional" properties of interest. Appendix I contains detailed specifications of the status of transactions and the transaction tree; we extract relevant pieces from them as needed.

\subsection{Specification Language: Larch}

The formal specification language used in this paper is Larch [9], though others such as VDM [3], Z [1], or OBJ [8], might also be suitable. Larch was designed to specify the functionality of sequential programs, in particular properties of abstract data types. A Larch specification has two parts: (1) A trait, written in an algebraic specification language, describes an object's intrinsic properties that are independent of the model of computation (e.g., elements in sets are unordered and not duplicated); (2) An interface, written in a predicative language using pre- and post-conditions, describes the effects on the object's state as operations are executed (e.g., its change in value or allocation of new storage). The advantage gained in using Larch is this explicit separation of concerns between state-independent and state-dependent behavior.

As applied to the transaction model of computation, we make three extensions to Larch interfaces for operations, as similarly made in [2].

- A when clause states the condition on the state of the system that must hold before the operation proceeds. Specifying this condition is often necessary since the state of the system may change between the point of invocation (when the pre-condition must hold) and the actual point of execution of the operation (when the when-condition must hold).

- We assume that each operation is operation-consistent, that is, an operation completes entirely or not at all. ${ }^{3}$ No intermediate states of an operation-consistent operation are observable between the state in which the when-condition holds and the state in which the post-condition holds. For an operation $o p$ that is a sequence of other operation-consistent operations $o p_{i}$ that may be interleaved with operations of other transactions and have observable effects, we specify $o p$ 's effects as a sequence of named operations $o p_{i}$ 's, each of which is specified as any operation-consistent operation. The only example of this kind of operation in this paper is the pause operation on subatomic objects found in Section 5.

- Self is used to denote the transaction invoking the operation.

Further details of Larch are provided as necessary. See [10] for a more complete discussion.

\footnotetext{
${ }^{3}$ Again, we use terminology from [5], but more standard terminology would call this property atomic, as in atomic operation or atomic action [2]. Since we use "atomic" for transactions, we needed to introduce a different term. Note that since a transaction is a sequence of operations, operation-consistency is a weaker property than transaction-consistency; it permits the partial effects of aborted transactions to be observed, while transaction-consistency does not.
} 


\section{Class Recoverable}

Conceptually, there are two kinds of storage for objects: volatile storage whose contents are lost upon crashes, and stable storage whose contents survive crashes with high probability. (Stable storage may be implemented using redundant hardware [13] or replication [4].) Recoverable objects are allocated in volatile storage, but their values are "logged" to stable storage so that recovery from crashes can be performed. If every recoverable object is written to stable storage after modifying operations are performed on it in volatile storage, then its state may be recovered after a crash. Restoring an object's state simply requires "replaying the log": redoing the effects of committed transactions and undoing the effects of aborted ones.

\subsection{Avalon Class Definition}

The programmer's interface to a recoverable object is through the Avalon/C++ class header shown in Figure 3-1.

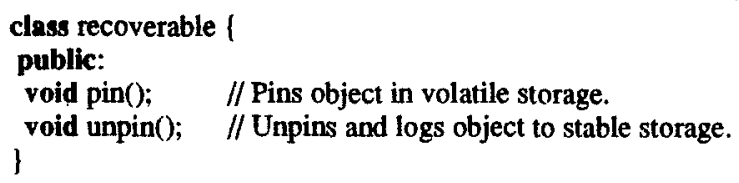

Figure 3-1: Avalon Class Recoverable

Informally, the pin operation causes the pages in volatile storage containing the object to be pinned; unpin causes the modifications to the object to be written to stable storage, and unpins its pages. A recoverable object must be pinned before it is modified, and unpinned afterwards. For example if $x$ is a recoverable object, a typical use of the pin and unpin operations within a transaction would be:

start $\{$ // begin transaction

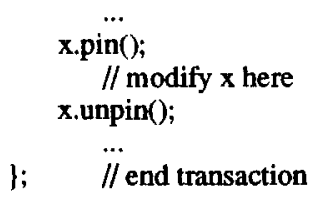

After a crash, a recoverable object is restored to a previous state in which it was not pinned. Transactions can make nested pin calls; if so, then the changes made within inner pin/unpin pairs do not become permanent, i.e., written to stable storage, until the outermost unpin is executed. Classes derived from recoverable inherit pin and unpin operations, which can be used to ensure persistence of objects of the derived class.

\subsection{Larch Specification}

The specification shown in Figure 3-2 captures the following three properties of recoverable objects:

1. Only one transaction can pin an object at once.

2. The same transaction can pin and unpin the same object multiple times.

3. Only at the last unpin does the object's value get written to stable storage. 

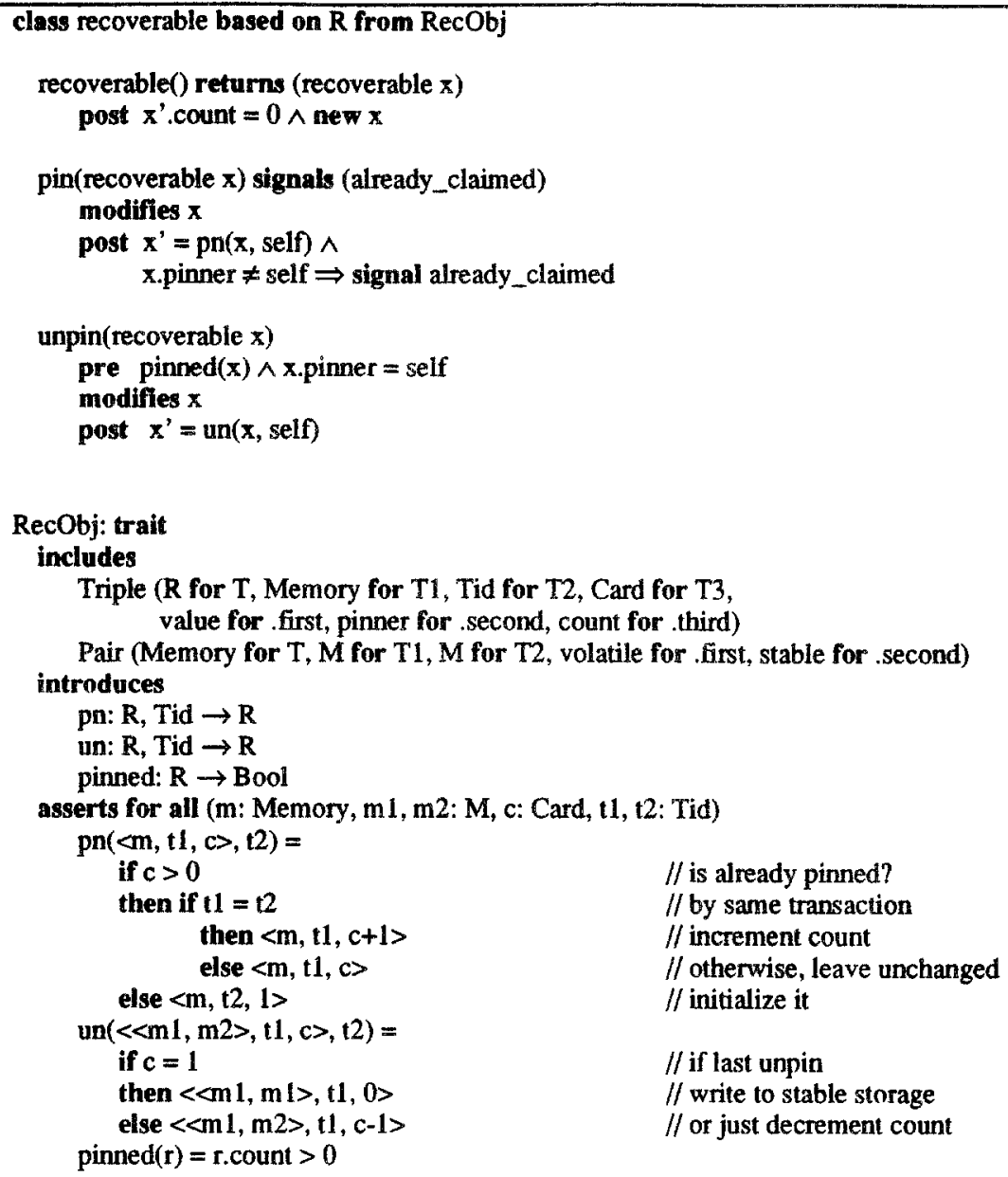

Figure 3-2: Larch Specification of Class Recoverable

We now walk through the specification in detail. The top part of the specification contains Larch interface specifications for a constnuctor given by the class name, recoverable. and the two operations. $\mathrm{pin}$ and unpin. ${ }^{4}$ The bottom part contains the Larch trait RecObj, which gives meaning to the assertion language of the interface specifications.

We see in RecObj that a recoverable object is "modeled" as its value in memory, a single transaction

\footnotetext{
${ }^{4}$ We depart from $\mathrm{C}++$ syntax for an operation's header by explicitly listing as an argument to each operation the implicit $\mathrm{C}++$ object this, and listing the returned object and its type in a returns clause.
} 
identifier, and a pin count:

Triple (R for T, Memory for T1, Tid for T2, Card for T3, value for .first, pinner for .second, count for third)

Here we include the trait Triple, defined in Appendix II, and rename (through the for clauses) the sort and functions identifiers it introduces. For example, $R$, the sort identifier introduced for recoverable objects, renames the sort identifier $T$ introduced in Triple. Memory itself is modeled as a pair of values, one each for volatile and stable storage:

Pair (Memory for T, M for T1, M for T2, volatile for .first, stable for .second)

The constructor's post-condition initializes the pin count to be zero and ensures that new storage is allocated for the returned object. An omitted pre-condition is interpreted as equivalent to pre true. Thus, the constructor can be called in any state.

Pin's post-condition specifies how the state of a recoverable object changes: $x$ stands for the object's initial state (upon invocation) and $x^{\prime}$ stands for its final state (upon return). Pin might terminate with an error condition signaled to the invoker to indicate that the object to be pinned is already pinned by some other transaction. Pin's post-condition makes use of the auxiliary function, $p n$, defined in the trait RecObj:

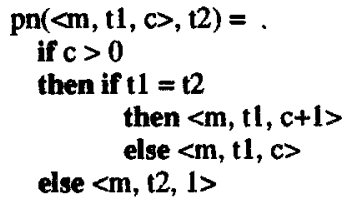

It takes a recoverable object's state (of sort $R$ ) and a transaction identifier (of sort Tid) and returns a (new) state for a recoverable object. If the count $(c)$ is non-zero, then the object must be pinned. If the object is pinned by a transaction $(t I)$ that is the same as the transaction $(t 2)$ attempting to pin the already pinned object, then the count is incremented; otherwise, the object is left unchanged. If the object is not already pinned, then its state is initialized with the pinning transaction's identifier and a count of 1 .

Unpin's pre-condition requires that an object cannot be unpinned unless it is already pinned; moreover it must be pinned by the calling transaction. $U n$ is defined as follows:

$$
\begin{aligned}
& \text { un }(<<\mathrm{m} 1, \mathrm{~m} 2>, \mathrm{t} 1, \mathrm{c}>, \mathrm{t} 2)= \\
& \text { if } \mathrm{c}=1 \\
& \text { then }<<\mathrm{m} 1, \mathrm{~m} 1>, \mathrm{t} 1,0> \\
& \text { else }<<\mathrm{m} 1, \mathrm{~m} 2>, \mathrm{t} 1, \mathrm{c}-1>
\end{aligned}
$$

Unlike for $p n$, it is unnecessary for $u n$ to check if the object is already pinned and if the transaction ( $t l$ ) that currently has the object pinned is the same as the unpinning transaction ( $t 2)$; unpin's pre-condition checks for this case. Un simply checks if there is only one outstanding call to pin $(c=1)$, in which case the value of the object in volatile storage is written to stable storage; otherwise, the count is decremented.

Both pin and unpin have a modifies clause, which lists the set of objects in the state of the entire system whose values may possibly change. It is a strong indirect assertion about which objects may not change in value. This assertion is implicitly conjoined to the operation's post-condition. An omitted modifies clause is equivalent to the assertion modifies nothing, meaning no objects are allowed to change in value. 


\subsection{Deriving From Class Recoverable}

A typical use of class recoverable is to define a derived class for ohjects that are intended to be persistent. For example, suppose we derive a new class, recov_int, from recoverable:

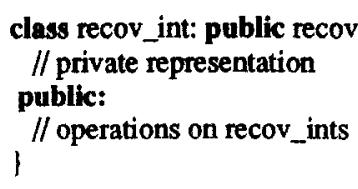

If Int is the sort identifier associated with values of recoverable integer objects, then the identifier $M$ that appears in the RecObj specification would be renamed with Int. The header for the Larch interface specification for the recov_int class would look like:

class recov_int based on $R$ from RecObj (Int for $M$ )

//... specifications of recov_int's operations ...

\section{Class Atomic}

The second base class in the Avalon/C++ hierarchy is atomic. Atomic is a subclass of recoverable, specialized to provide two-phase read/write locking and automatic recovery. Locking is used to ensure serializability, and an automatic recovery mechanism for objects derived from atomic is used to ensure transaction-consistency. Persistence is "inherited" from class recoverable since pin and unpin are inherited through $\mathrm{C}++$ inheritance.

\section{I. Avalon Class Definition}

Figure 4-1 gives the class header for atomic.

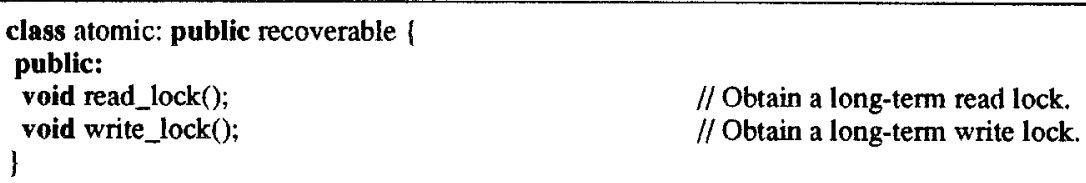

Figure 4-1: Avalon Atomic Class

Atomic objects should be thought of as containing long-term locks. Under certain conditions, read_lock (write lock) gains a read lock (write lock) for its caller. Transactions hold locks until they commit or abort. Read_lock and write_lock suspend the calling transaction until the requested lock can be granted, which may involve waiting for other transactions to complete and release their locks. If read_lock or write_lock is called while the calling transaction already holds the appropriate lock on an object, it retums immediately.

\subsection{Larch Specification}

Figure 4-2 gives the Larch interfaces and trait for class atomic. As indicated in the trait AtomObj, an atomic object is a recoverable object, along with a set of transactions that hold read locks on the object and a set of transactions that hold write locks on it: 


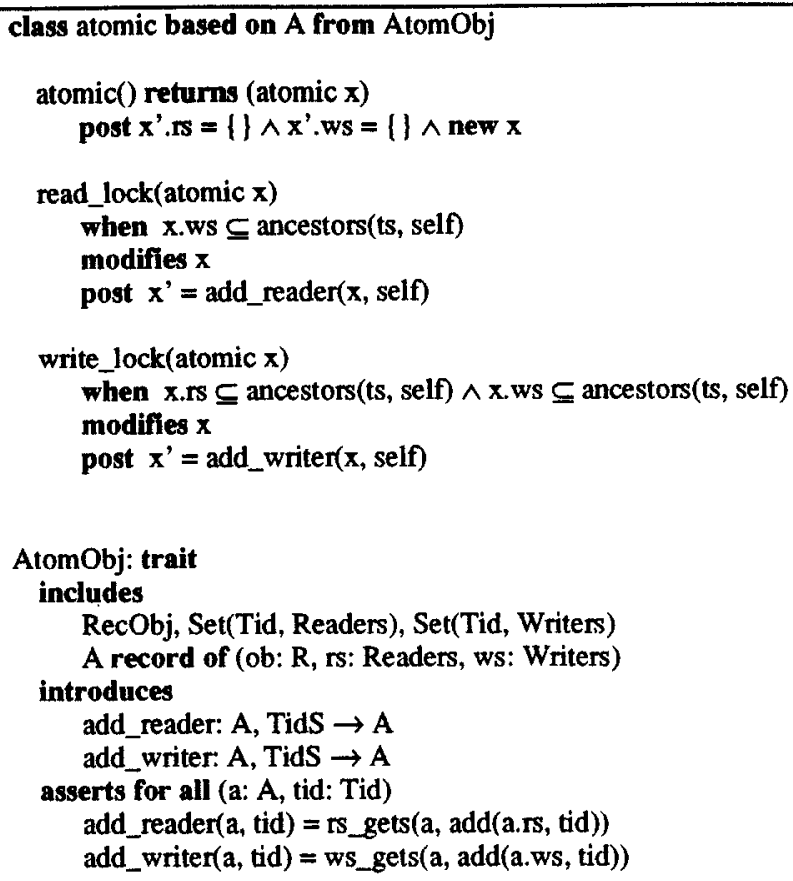

Figure 4-2: Larch Specification of Class Atomic

A record of (ob: $R$, rs: Readers, ws: Writers)

Even though only one writer can be modifying the state of an atomic object at once, we keep track of a set of transactions with write locks because a child transaction can get a write lock if its parent has one. The constructor for atomic initializes both the sets of readers and writers to be empty.

The transaction tree $t s$ of type tidTree is global information:

class tidTree based on TransIdS from TransIdTree

// ... TransIdTree defined in Appendix I ...

global ts: tidTree

Appendix I gives traits for defining a transaction tree. providing functions like ancestors, which returns the set of transactions that are ancestors of a given transaction (including itself). We declare the transaction tree global only for convenience since such objects could be passed as explicit arguments 10 each operation.

Read_lock's when-condition states that a transaction can get a read lock if all transactions holding write locks are ancestors; write_lock's when-condition states that a transaction can get a write lock if all transactions holding read or write locks are ancestors. These two requirements reflect the conditions of Moss's locking rules for nested transactions [14], which are implemented in Avalon/C++. 
As usual, the post-conditions look simple; the trait's add_reader and add_writer functions do the actual work, by adding the calling transaction to the appropriate set. Notice that since $r s(w s)$ is a set, adding a transaction that already is in it has no effect. Thus, if the calling transaction already has a read (write) lock on the object, no change is made; otherwise, it obtains a read (write) lock.

\subsection{Deriving From Class Atomic}

Suppose we now define an atomic int class as follows:

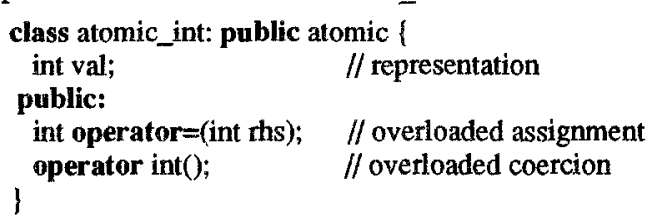

As for the previous recov_int example, when giving the Larch interface specification for the atomic_int class, we rename the sort identifier $M$, introduced in the RecObj trait and included in the AtomObj trait, :

class atomic_int based on A from AtomObj (Int for M)

$/ / \ldots$ specifications of atomic_int's operations ...

Now let us specify atomic_int's coercion operation, which takes an atomic_int, $x$, and returns a regular $C++$ int, $i$ :

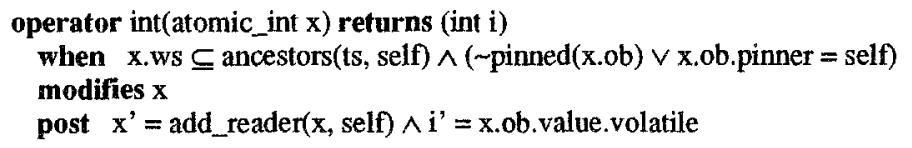

The second conjunct of the post-condition makes the climactic point: The value (of sort Int) of the int object $i$ refurned is the value (of sort Int) in volatile storage of the recoverable object component of the atomic_int $x$. We retrieve the value from volatile storage because we can assume that the when-condition held: if the object is pinned, but not yet unpinned (by self) then we want $x$ 's most recent value; if the object is unpinned, then the values in volatile and stable storage would be identical.

Let us examine how the derived class uses the inherited operations, relying on their specifications. An Avalon/C++ implementator of atomic_int can use write_lock and read_lock of class atomic and pin and unpin of class recoverable to ensure the serializability, transaction-consistency, and persistence of atomic_ints. (Thus, atomic_int class's clients can assume these properties hold for all atomic_ints.) For example, here is how the coercion operation would be implemented in Avalon/C++:

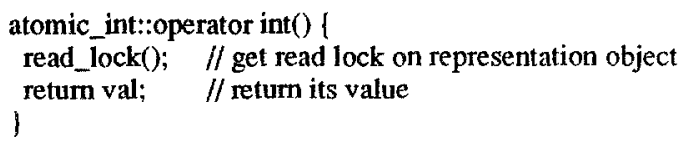

Using the specification of class atomic's read lock operation, we can show (1) the coercion operation's when-condition trivially implies read_lock's when-condition; and (2) read_lock's post-condition guarantees the calling transaction has a read lock on the atomic_int object. These two properties imply that $\mathrm{val}$, the int representation of an atomic_int will not be read and returned until the calling transaction obtains a read lock on the atomic_int, and moreover, no concurrent transactions have write locks on it. 


\section{Class Subatomic}

The third, and perhaps most interesting, base class in the Avalon/C++ hierarchy is subatomic. Like atomic, subatomic provides the means for objects of its derived classes to ensure atomicity. While atomic provides a quick and convenient way to define new atomic objects, subatomic provides more complex primitives to give programmers more detailed control over their objects' synchronization and recovery mechanisms by exploiting type-specific properties of objects. For example, a queue object with enqueue and dequeue operations can permit enqueuing and dequeueing transactions to go on concurrently, even though those transactions are both "writers." In defining an atomic_queue class by deriving from class atomic, such concurrency would not be possible; deriving from class subatomic makes it possible. See [5] for details and other examples.

\subsection{Avalon Class Definition}

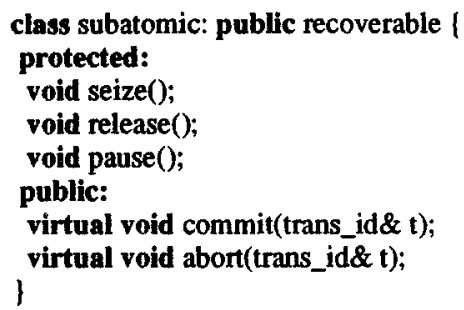

\author{
// Gains short-term lock. \\ // Releases short-term lock. \\ // Temporarily releases short-term lock. \\ // Called after transaction commit. \\ // Called after transaction abort.
}

Figure 5-1: Avalon Subatomic Class

A subatomic object must synchronize concurrent accesses at two levels: short-term synchronization ensures that concurrently invoked operations are executed in mutual exclusion, and long-term synchronization ensures that the effects of transactions are serializable. Short-term synchronization is used to guarantee operation-consistency of objects derived from subatomic.

Subatomic provides the seize, release, and pause operations for short-term synchronization. Each subatomic object contains a short-term lock, similar to a monitor lock or semaphore. Only one transaction may hold the short-term lock at a time. The seize operation obtains the short-term lock, and release relinquishes it. Pause releases the short-term lock, waits for some duration, and reacquires it before returning. Thus, these operations allow transactions mutually exclusive access to subatomic objects. Seize, release, and pause are protected members of the subatomic class since it would not be useful for clients to call them.

To ensure transaction-consistency, subatomic provides commit and abort operations. Whenever a toplevel transaction commits (aborts), the Avalon/C++ run-time system calls the commit (abort) operation of all objects derived from subatomic accessed by that transaction or its descendants. Abort operations are also called when nested transactions "voluntarily" abort. Since commit and abort are C+t virtual operations, classes derived from subatomic are expected to reimplement these operations. Thus, subatomic allows type-specific commit and abort processing, which is useful and often necessary in implementing user-defined atomic types efficiently. 


\subsection{Larch Specification}

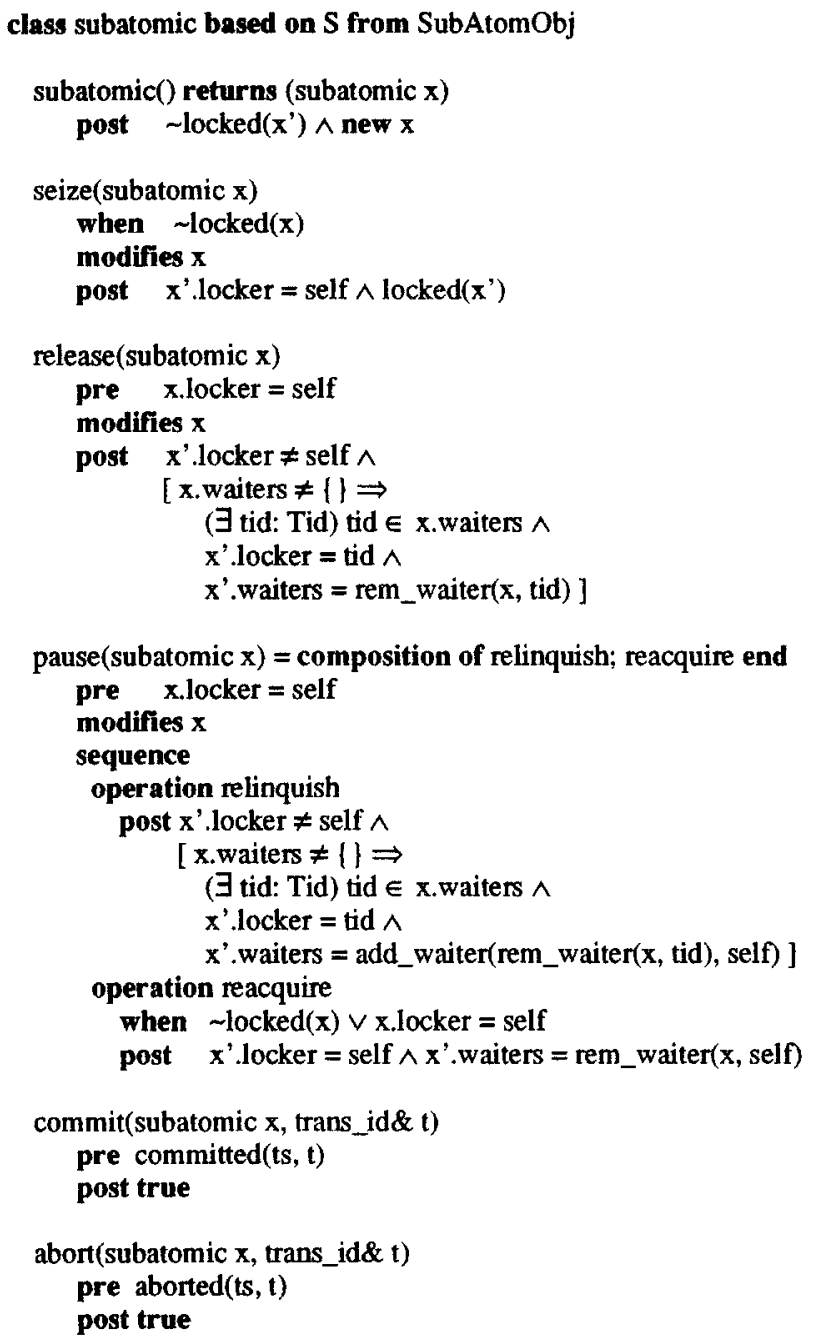

Figure 5-2: Larch Specification of Class Subatomic (Interfaces)

Figures 5-2 and 5-3 give the Larch interfaces and trait for class subatomic. As indicated in the trait SubAtomObj, a subatomic object is a recoverable object, along with the transaction holding the short-term lock, and a set of transactions that are waiting to acquire it.

$S$ record of (ob: $R$, locker: Tid, waiters: Waiters)

Initially, as specified in the constructor, no one holds the short-term lock on the object. 


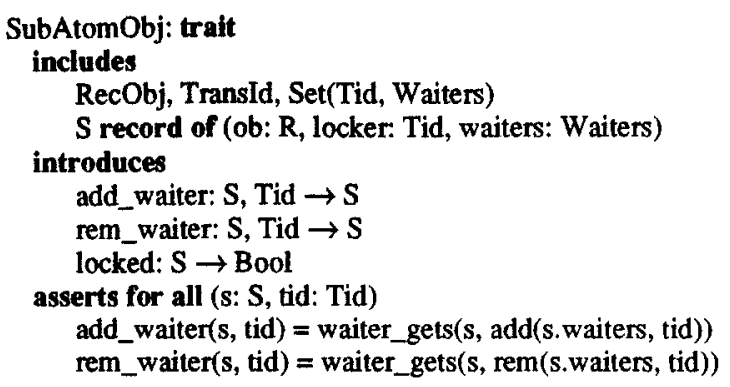

Figure 5-3: Larch Specification of Class Subatomic (Trait)

Seize's when-condition states that a transaction must wait until no transaction holds the short-term lock on the object before acquiring the lock. The post-condition states that the calling transaction obtains the short-term lock on the object, and the object is now locked.

Release's pre-condition requires that the calling transaction be the one who has the lock on the object. The post-condition states that the caller will no longer have the lock upon return, and if some other transaction is waiting to obtain the lock, it is given the lock.

Pause's pre-condition is similar to release's. The rest of its specification, however, is unlike all others. Pause's effects are specified in terms of a sequence of two operations, each of which can be interleaved with other operations of other transactions. First, pause relinquishes the short-term lock as release does. However, relinquish's post-condition differs from release's in one critical way: the calling transaction is added to the waiting set of transactions upon relinquishing the lock. The second operation, reacquire, in the sequence is delayed until either no one has a lock on the object or some other transaction has released the lock and given it back to self. Its post-condition ensures that the original caller of pause again possesses the short-term lock upon return.

The specifications of commit and abort deserve special attention. Each is called with a trans_id argument denoting some transaction that has committed (aborted) in the given (global) transaction tree ts. The implicit "modifies nothing" assertion states that no change to the object is allowed. This seemingly strong assertion reflects the intention that commit and abort operations are to have only "benevolent" side effects on the object's state, meaning that the abstract state of the object remains the same, though the representation state may change. Indeed, typical Avalon/C++ implementations of commit operations simply discard redundant state information stored in the representation object. not affecting the abstract state at all. Typical implementations of abort operations use this redundant state information to unito tentative changes performed by the aborting transaction (and any of its descendants that have committed with respect to it).

Deriving from class subatomic is similar to deriving from class recoverable or class atomic and is omitted for brevity. 


\section{Observations}

\subsection{About Avalon}

The exercise of formally specifying the Avalon/C++ classes revealed unstated assumptions about the actual implementation and made more precise Avalon/C++'s fundamental semantics.

One unstated assumption in the underlying operating system (Camelot) is reflected in the implementation, but was never made explicit until we wrote the formal specification of class recoverable. The Avalon/C++ implementation precludes the possibility of concurrent pins by different transactions; Camelot forbids this situation because it assumes that any transaction that pins an object intends to modify it. This assumption is one example of where crash recovery and concurrency cannot be separated when reasoning about Avalon programs. Without concurrency, one can give a meaning to persistence; without crash recovery, one can give a meaning to the correct synchronization of processes. But to support both, there are points where one must consider both persistence and synchronization together.

Another kind of unstated assumption discovered from this exercise is implicit pre-conditions. For example, whereas pin has no pre-condition, unpin does. This assymmetry in the specifications reflects the assymmetry that exists in the actual implementation. An earlier version of the specification of unpin did not have a pre-condition, but not until the implementor was shown this (incorrect) version was the unstated pre-condition revealed. In fact, upon seeing the assymmetry in the current version of the specification, the implementor realized that the pre-condition on unpin could easily be removed by performing a run-time check, as is already done for pin. ${ }^{5}$

Specifying the class atomic helped make the rules for obtaining long-term locks more precise. It also makes explicit, by modeling a set of writers, not just a single writer, the property that more than one transaction might hold a long-term write lock on an object at once. Recall this situation can arise because of nested transactions. On the other hand, the specification of class subatomic makes explicit that only one transaction (the locker) can have the short-term mutual exclusion lock on an object at once.

Specifying the class subatomic helped identify a subtle source of a potential deadlock situation. As specified in Figure 5-2, if there are waiters, pause will not return until some transaction, tid, other than the calling one, self, grabs the short-term lock and returns, thereby releasing the lock. If tid does not return (perhaps it is waiting for some synchronization condition to become true), then self will not be able to return since it will be unable to reacquire the lock. In fact, this situation can arise in the current Avalon/C++ implementation and was discovered only through trial and error when debugging some simple examples. Had we done the specification beforehand, we could more easily have anticipated this problem.

\subsection{About Larch}

In the traditional spirit of Larch, all the complexity of a specification is relegated to the traits. The rule-of-thumb is: If the post-condition becomes unwieldy then introduce a trait function to capture the intended property. However, one place where that cannot easily be done is in specifying nondeterminism. Since traits define (deterministic) functions, interfaces are responsible for specifying nondeterministic

\footnotetext{
${ }^{5}$ The astute reader may have noticed that un's second argument, a vestige of the earlier specification, was ignored in its definition; if the pre-condition for unpin is removed, then the second argument is necessary.
} 
behavior. For example, the use of the existential operator in the post-conditions of release and pause is unavoidable.

Not surprisingly, Larch needed to be extended to deal with concurrency, as exemplified here for Avalon/C++ and in [2] for Modula-2+. The two most important extensions are: (1) the need to specify an operation's effects through the specification of a sequence of other operations, and (2) the when clause used for stating a third kind of condition in addition to pre- and post-conditions. As an aside, this when-condition influenced the Avalon/ $\mathrm{C}++$ designers who added a when statement to the language. This statement, which makes appropriate calls to seize, release, and pause, is akin to a conditional critical region.

One critical class of properties that cannot be stated in Larch, even as currently extended, is liveness. For example, one cannot say that an object's commit or abort operation will eventually be called. Unfortunately, many programs may be correct with respect to safety but can deadlock or livelock in practice. In particular, typical implementations of operations of classes derived from subatomic test at run-time whether some transaction has committed; obtaining the short-term lock often depends on this test to succeed. So, sometimes no progress can be made until some transaction has committed. We have seen in the previous section where deadlock may arise in the implementation, and how the specification permits for this behavior. Though Larch was never intended to address liveness properties, in the context of concurrent transactions, such properties are important to state for practical reasons.

\section{Final Remarks}

The specifications presented here represent ongoing work. They continue to change as we continue to specify more of Avalon/C++'s intricacies, such as: (1) Avalon/C++'s transaction model of state, which must include two kinds of store, volatile and stable. It must also include the entire transaction tree, the status of each transaction in the tree, and the sets of locks each transaction holds. (2) System-wide commit and abort operations, which must be defined on behalf of a transaction committing or aborting. For example, the system-wide commit operation would take a transaction identifier and a timestamp, modifying the status of some transaction in the transaction tree. (3) A system-wide recover operation, which would define the effects of recovering from a crash. We would need to modify the specification for a recoverable object by keeping track of the entire history of operations performed on it in order to capture the set of possible values such an object can have [11]. (4) Avalon/C++'s built-in class, trans_id, which has operations for creating transaction identifiers and testing whether two transactions are serialized with respect to each other. Appropriate trait functions would be added to the trait TransIdTree of Appendix I to facilitate the specification of trans $i d$.

As we generate these specifications, we would also like to prove theorems about the objects being specified. For example, from the specification in Figures 5-2 and 5-3 we can prove that the transaction (tid) given the lock upon return from release is different from the calling transaction (self). The proof of this property depends on the following property of subatomic objects: $(\forall x: S)$ x.locker $\notin$ waiters $(x)$. Our plan is to use the Larch Prover [7] to help with these proofs.

Though the specification of Avalon/C++ is incomplete, we have specified a critical piece of it since all user-defined classes derive from the built-in ones. Knowing early on that a fundamental part of Avalon/C++'s semantics is implemented correctly is a tremendous reassurance to us as Avalon implementors as well as to all Avalon programmers. In conclusion, writing the formal specifications of Avalon/C++'s built-in classes has helped clarify places in the language where features interact, make 
explicit unstated assumptions, and make precise complex non-functional properties of objects.

\section{Acknowledgments}

Discussions with John Guttag and Jim Horning and the examples given in [2] inspired my on-the-fly interface language design, in particular the Larch extensions for concurrency. Chun Gong helped develop the traits and Rick Lerner helped check the interfaces. I am grateful to all members of the Avalon group. in particular, Maurice Herlihy and David Detlefs, who helped design Avalon/C++, and David who was instrumental in building it.

\section{Transactions and the Transaction Tree}

Below is a Larch trait that specifies a transaction's state. We assume the existence of a TimeStamp trait used for generating timestamps of sort Time, and a UniqueId trait used for generating unique identifiers of sort $I d$. A transaction can be either committed, active, or aborted. Only committed transactions are given timestamps.

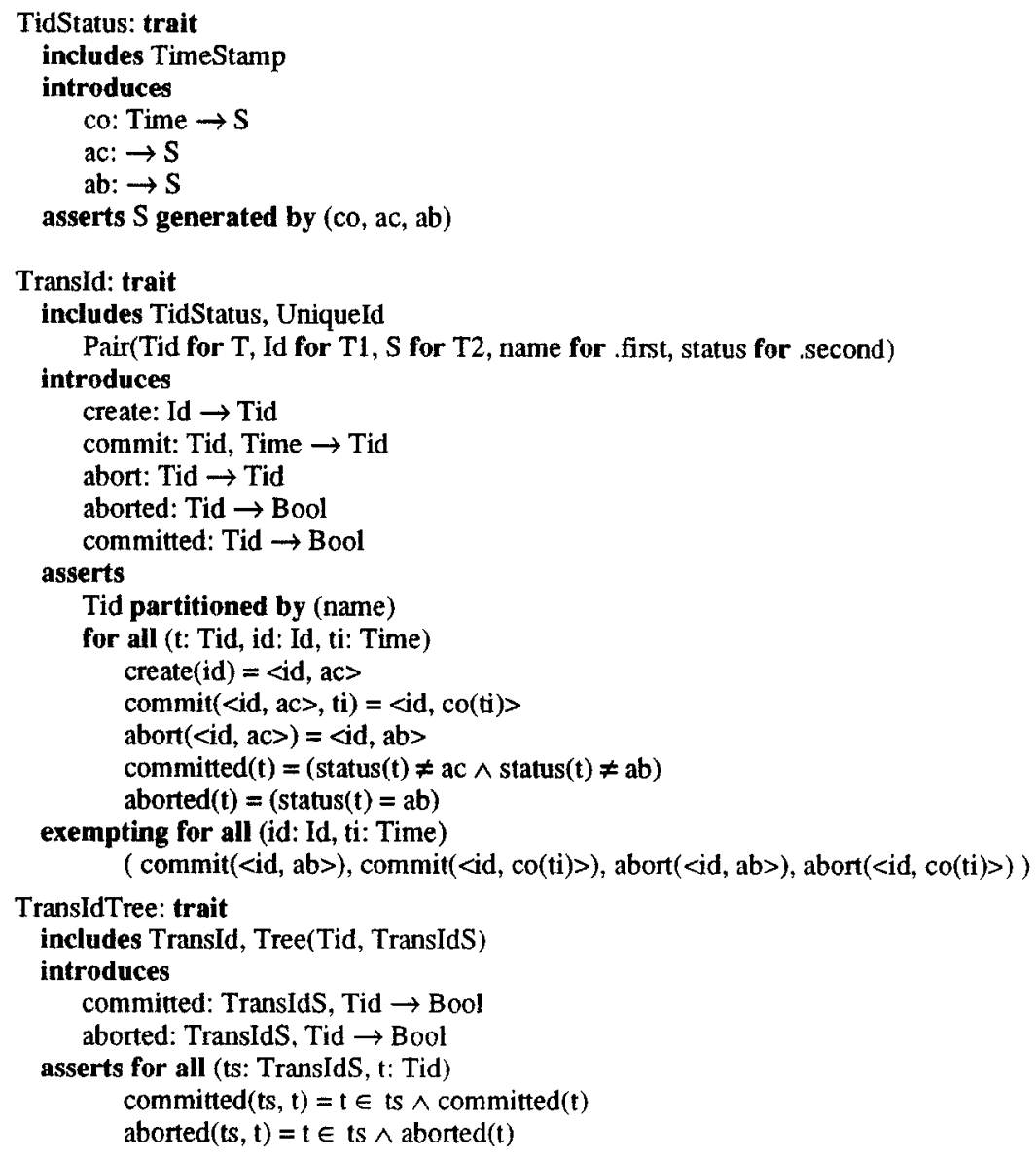




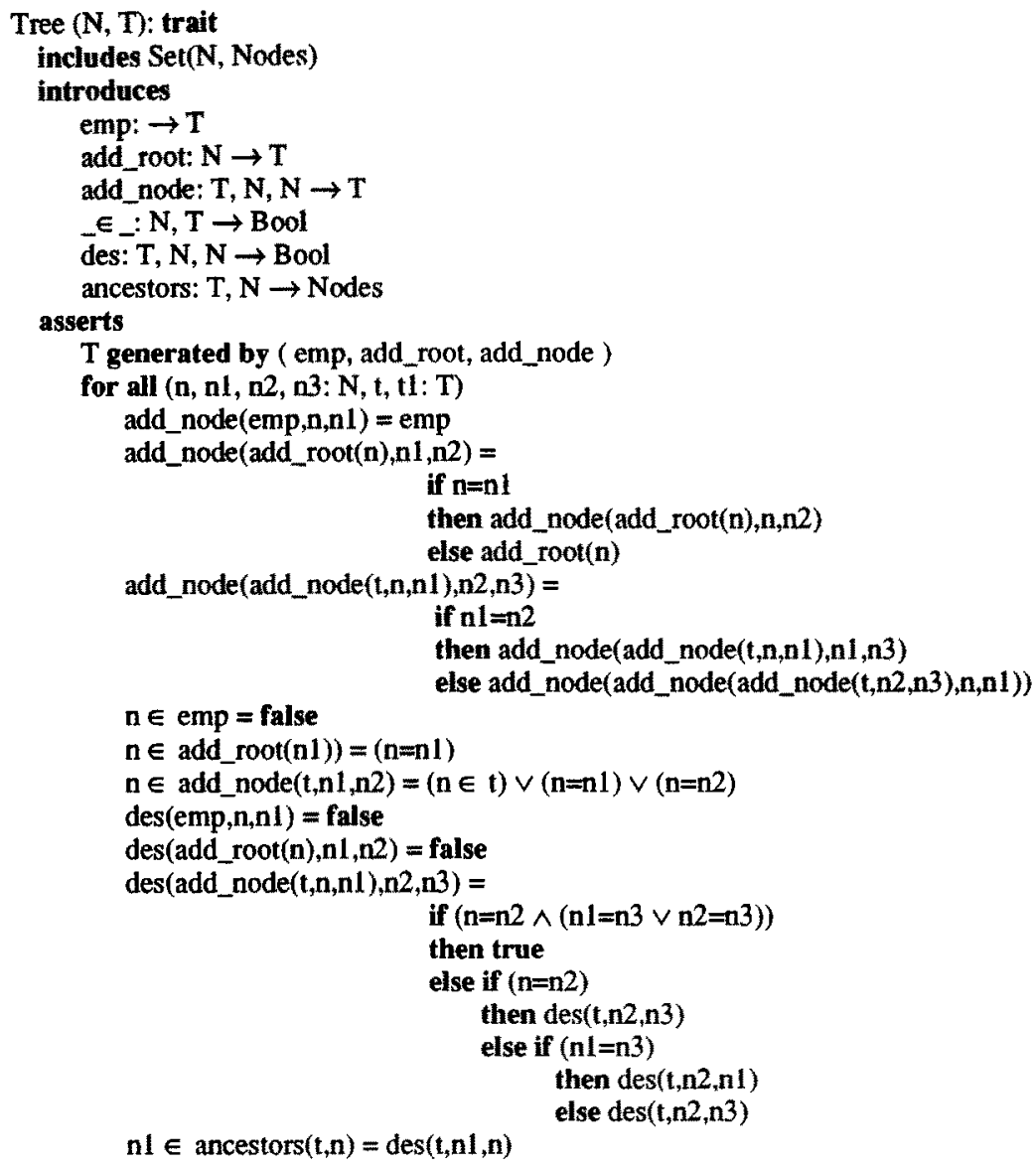

\section{Auxiliary Traits}

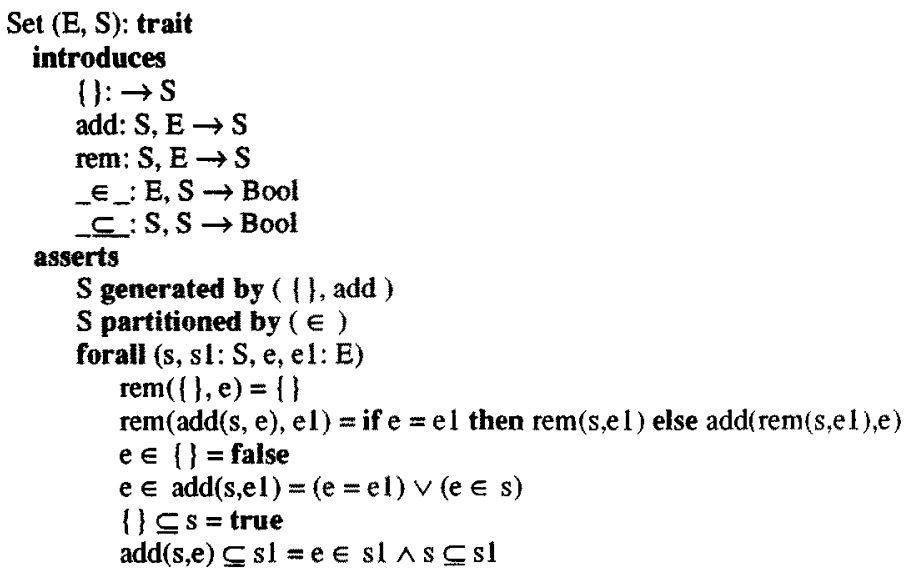




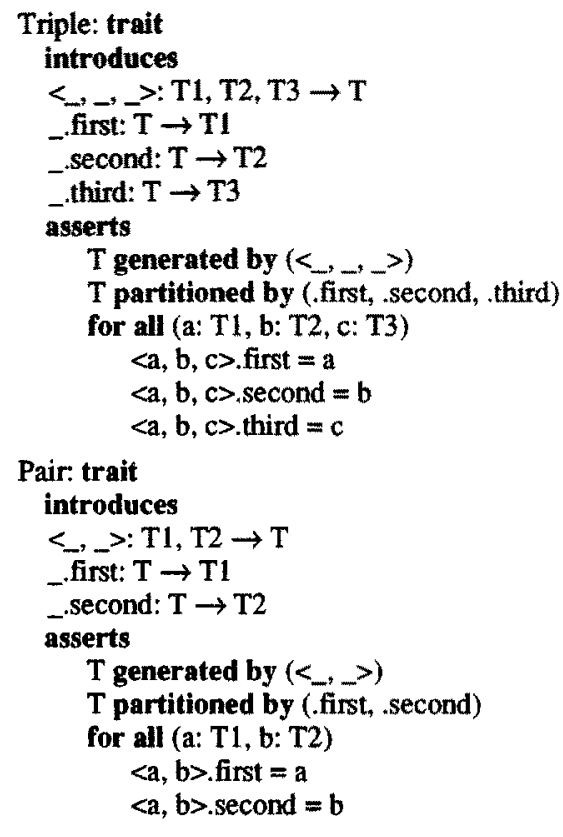

Records are a shorthand for a trait defined as follows. For each record of the form

$$
S \text { record of }\left(f_{1}: S_{1}, \ldots, f_{n}: S_{n}\right)
$$

Append to the function declarations of the enclosing trait:

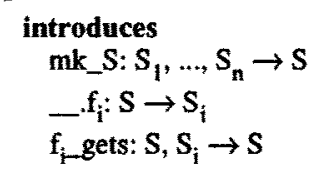

for $1 \leq \mathrm{i} \leq \mathrm{n}$.

Append to the set of equations of the enclosing trait:

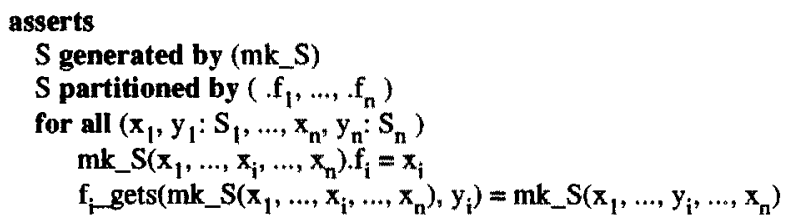

for $1 \leq \mathrm{i} \leq \mathrm{n}$. 


\section{References}

[1] J.R. Abrial.

The Specification Language Z: Syntax and Semantics.

Technical Report, Programming Research Group, Oxford University, 1980.

[2] A. Birrell, J. Guttag, J. Horning, R. Levin.

Synchronization Primitives for a Multiprocessor: A Formal Specification.

In Proceedings of the Eleventh ACM Symposium on Operating Systems Principles, pages 94-102.

ACM/SIGOPS, 1987.

[3] D. Bjomer and C.G. Jones (Eds.).

Lecture Notes in Computer Science. Volume 61: The Vienna Development Method: the Meta-language.

Springer-Verlag, Berlin-Heidelberg-New York, 1978.

[4] D. S. Daniels.

Distributed Logging for Transaction Processing.

In Proceedings of the 1987 ACM Sigmod International Conference on Management of Data. Association for Computing Machinery, San Francisco, CA, May, 1987.

[5] D. L. Detlefs, M. P. Herlihy, and J. M. Wing.

Inheritance of Synchronization and Recovery Properties in Avalon/C++.

IEEE Computer :57-69, December, 1988.

[6] K. P. Eswaran, J. N. Gray, R. A. Lorie, and I. L. Traiger.

The Notions of Consistency and Predicate Locks in a Database System.

Communications of the ACM 19(11):624-633, November, 1976.

[7] S.J. Garland and J.V. Guttag.

Inductive Methods for Reasoning about Abstract Data Types.

In Proceedings of the 15th Symposium on Principles of Programming Languages, pages 219-228. January, 1988.

[8] J.A. Goguen and J.J. Tardo.

An Introduction to OBJ: A Language for Writing and Testing Formal Algebraic Program Specifications.

In Proceedings of the Conference on Specifications of Reliable Software, pages 170-189. Boston, MA, 1979.

[9] J.V. Guttag, J.J. Horning, and J.M. Wing.

The Larch Family of Specification Languages.

IEEE Software 2(5):24-36, September, 1985.

[10] J.V. Guttag, J.J. Horning, and J.M. Wing.

Larch in Five Easy Pieces.

Technical Report 5, DEC Systems Research Center. July. 1985.

[11] M.P. Herlihy and J.M. Wing.

Reasoning About Atomic Objects.

Technical Report CMU-CS-87-176, Carnegie Mellon University Department of Computer Science, November, 1987.

[12] L. Lamport.

Time, clocks, and the ordering of events in a distributed system.

Communications of the ACM 21(7):558-565, July, 1978. 
[13] B. Lampson.

Atomic transactions.

Lecture Notes in Computer Science 105. Distributed Systems: Architecture and Implementation. Springer-Verlag, Berlin, 1981, pages 246-265.

[14] J.E.B. Moss.

Nested Transactions: An Approach to Reliable Distributed Computing.

Technical Report MIT/LCS/TR-260, Massachusetts Institute of Technology Laboratory for Computer Science, April, 1981.

[15] A. Spector, J. Bloch, D. Daniels, R. Draves, D. Duchamp, J. Eppinger, S. Menees, D. Thompson. The Camelot Project.

Database Engineering 9(4), December, 1986.

[16] B. Stroustrup.

The $\mathrm{CH}$ + Programming Language.

Addison-Wesley, Reading, Massachusetts, 1986.

[17] W.E. Weihl.

Specification and Implementation of Atomic Data Types.

PhD thesis, MIT, 1984. 